\title{
Extramedullary hematopoiesis in the absence of myeloproliferative neoplasm: Mayo Clinic case series of 309 patients
}

\author{
N. Fan', S. Lavu (1) ', C. A. Hanson² and A. Tefferi ${ }^{1}$
}

Extramedullary hematopoiesis (EMH) implies the production of erythroid and myeloid progenitor cells outside of the bone marrow. EMH in adults is typically seen in patients with myeloproliferative neoplasms (MPNs) but its association also with other conditions, including thalassemia, has long been recognized ${ }^{1}$. In both MPN and non-MPN settings, the liver and spleen are the two most frequent sites of EMH and it has been hypothesized that circulating hematopoietic cell filtration (entrapment), possibly via endothelial cell expressed ligands, such as chemokine ligand 12, rather than splenic stroma account for the particular phenomenon ${ }^{2-4}$; the concordant detection of MPN-specific mutations, such as JAK2V617F, and specific cytogenetic abnormalities in both bone marrow and splenic tissue of affected patients, supports this contention ${ }^{5,6}$.

Circulating hematopoietic progenitors mobilized as a result of otherwise nonspecific hematopoietic stressors have also been implicated in seeding non-hepatosplenic EMH (NHS-EMH) ${ }^{7}$. The latter has been reported in a variety of organs, including the central nervous system ${ }^{8}$, ovaries and tubes ${ }^{9}$, the $\operatorname{skin}^{10}$, the lungs and pleura ${ }^{11}$, the pericardium $^{12,13}$, lymph nodes ${ }^{14}$, and other sites. In a previously published report of 27 Mayo Clinic cases of NHS-EMH diagnosed antemortem between 1975 and $2002^{15}$, the most common associated condition was myelofibrosis and the most frequent involved site the thoracic vertebral column. The current study focuses on the Mayo Clinic experience with EMH cited in the absence of MPN. The objectives were to systematically

\footnotetext{
Correspondence: A. Tefferi (tefferi.ayalew@mayo.edu)

${ }^{1}$ Division of Hematology, Department of Medicine, Mayo Clinic, Rochester, MN, USA

2Division of Hematopathology, Department of Laboratory Medicine, Mayo Clinic, Rochester, MN, USA
}

describe associated conditions and involved sites and identify "idiopathic" cases and review their management and long-term outcome.

After approval by the Mayo Clinic institutional review board, institutional databases were screened through the Mayo Clinic Advanced Cohort Explorer (ACE) Tool, in order to identify patients with EMH. ACE is a clinical data repository maintained by the Unified Data Platform; ACE is enriched with multiple source patient information including patient demographics, diagnosis, hospital notes, laboratory reports, flowsheets, pathology reports, and clinical notes. With ACE's text search functionality, we queried "extramedullary hematopoiesis" or "EMH". We performed a retrospective database review of all identified patients between 1975 and 2018. Demographic, biochemical, genetic, radiological, and pathological data were collected and reviewed.

ACE identified 1933 cases of "EMH". Extensive review confirmed the absence of associated MPN in 336 cases. Among these, 27 cases involved pathology remarks during tissue biopsy for liver transplant and were excluded from further analysis. The most frequent associated conditions in the remaining 309 cases (Table 1) included myelodysplastic syndromes (MDS) $(n=41 ; 13 \%)$; acute myeloid leukemia (AML) $(n=28 ; 9 \%)$; hemolytic anemia $(n=24$; $8 \%)$; thalassemia $(n=22 ; 7 \%)$; non-Hodgkin's lymphoma (NHL), with excess cases with splenic marginal zone lymphoma $(n=19 ; 6 \%)$; immune thrombocytopenic purpura (ITP) $(n=17 ; 6 \%)$; metastatic cancer, with breast cancer being the most frequent $(n=17 ; 6 \%)$; plasma cell neoplasms, including polyneuropathy, organomegaly, endocrinopathy, monoclonal protein, and skin changes $(n=12 ; 4 \%)$; hereditary spherocytosis $(n=8 ; 3 \%)$; cirrhosis $(n=7 ; 2 \%)$; acute lymphoblastic leukemia $(n=6$; $2 \%)$; chronic lymphocytic leukemia $(n=6 ; 2 \%)$; Hodgkin's 
Fan et al. Blood Cancer Journal (2018)8:119

Page 2 of 4

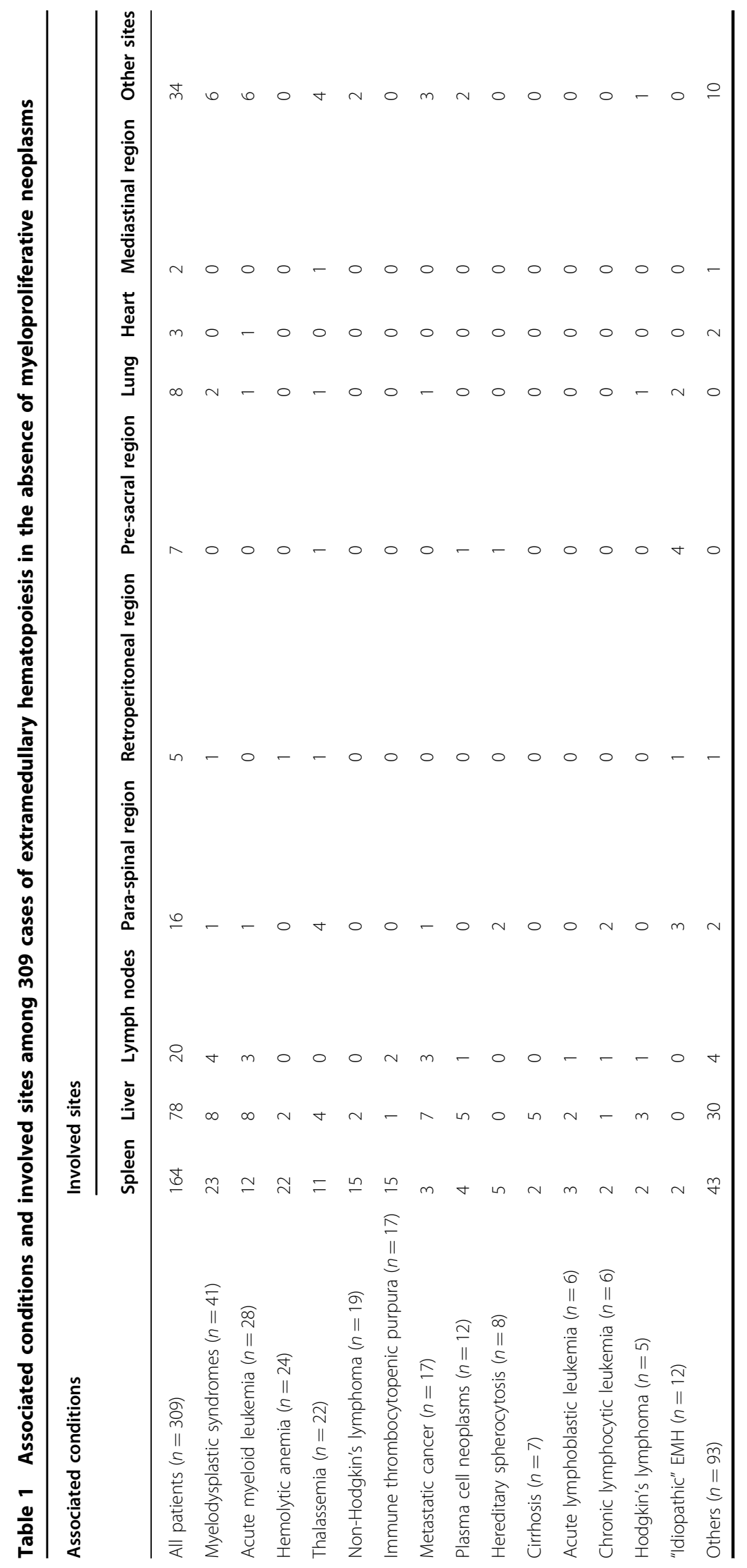

Blood Cancer Journal 


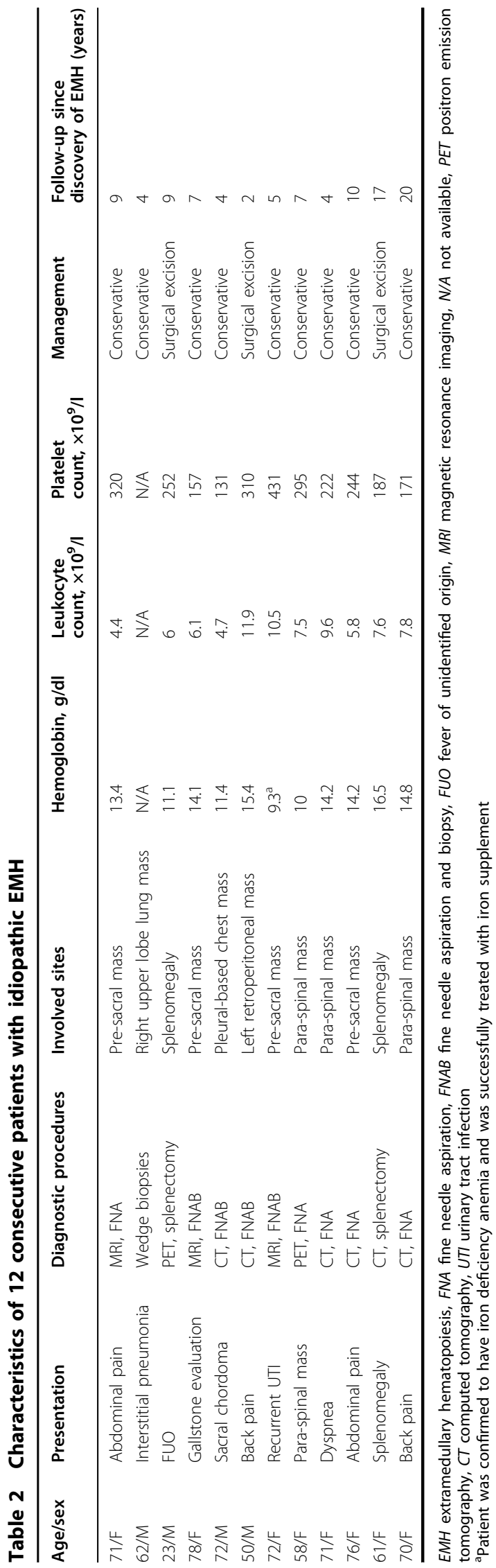

lymphoma $(n=5 ; 2 \%)$; and a spectrum of other hematologic and non-hematologic conditions with less than five incident cases, including large granular lymphocyte and natural killer cell disorders, chronic myelomonocytic leukemia, hemophagocytic lymphohistiocytosis, anemia of chronic disease, bone marrow failure syndrome, and fungal or viral infection including human immunodeficiency virus and cytomegalovirus; in 12 (4\%) cases, no overt associated condition was evident and the cases were accordingly assigned "idiopathic" EMH (further elaborated below). The most frequently involved sites included the spleen $(n=164 ; 53 \%)$, liver $(n=78 ; 25 \%)$, lymph nodes $(n=20 ; 6 \%)$, and the para-spinal region $(n=16$; $5 \%$ ) (Table 1). Other sites with lower number of incident cases included the pre-sacral, retroperitoneal, and mediastinal regions, the skull, maxillary region, the skin, kidneys, adrenal tissue, thyroid gland, ovaries, lung, heart, pleura, and pericardium.

A diagnosis of "idiopathic" EMH was established in 12 (4\%) patients (median age 71 years, range $23-78 ; 50 \%$ females). Most of these cases presented with nonspecific symptoms including abdominal and back pain and the EMH was an incidental discovery (Table 2); 1 patient with splenic EMH presented with fever of unidentified origin (FUO). Involved sites in the 12 patients with idiopathic EMH included 4 pre-sacral, 3 para-spinal, 2 spleen, and 1 each retroperitoneal, pleural-based chest mass, and right upper lobe lung mass. All idiopathic EMH cases were evaluated with imaging studies and diagnosis was subsequently confirmed by pathology review. Past medical history was non-contributory. Complete blood counts were normal in 7 cases, showed anemia in 4 cases, and were not available in 1 case (Table 2). The median followup time since the discovery of idiopathic EMH was 7 years (range 2-20). None of the patients with idiopathic EMH showed evidence of any malignancy, including MPN or other hematologic disorders, either at presentation or during follow-up. Nine (75\%) of the 12 patients with idiopathic EMH were managed conservatively; the 2 patients with splenic EMH underwent splenectomy and 1 patient had surgical excision of the EMH mass in order to prevent compression of the ureter. The FUO in the patient with splenic EMH resolved with splenectomy.

We present the largest experience in EMH without associated MPN, in adults. We confirm the spleen and liver being by far the most frequent organs involved; MDS, AML, hemolytic anemia, thalassemia, NHL, ITP, metastatic cancer, and plasma cell neoplasms constituted the most frequent associated conditions. Considering the lack of information, we were particularly interested in "idiopathic" EMH and its natural history. We identified 12 cases of idiopathic EMH, which often represented an incidental discovery during evaluation of unrelated symptoms. None of the patients with idiopathic EMH 
harbored occult malignancies or subsequently developed MPN or other myeloid malignancies. Accordingly, our observations do not support undertaking extensive investigations targeting MPN or other malignancies in idiopathic EMH and simple monitoring might be adequate.

\section{Author contributions}

All authors have reviewed the manuscript and gave their approval. A.T. designed the study, contributed patients, helped abstract patient information, and wrote the paper. N.F. abstracted patient information and prepared manuscript tables. S.L. helped with study design and data retrieval. C.A.H. was in charge of information on pathology.

\section{Conflict of interest}

The authors declare that they have no conflict of interest.

\section{Publisher's note}

Springer Nature remains neutral with regard to jurisdictional claims in published maps and institutional affiliations.

Received: 17 August 2018 Accepted: 21 September 2018

Published online: 19 November 2018

\section{References}

1. Sorsdahl, O. S., Taylor, P. E. \& Noyes, W. D. Extramedullary hematopoiesis, mediastinal masses, and spinal cord compression. JAMA 189, 343-347 (1964).

2. O'Keane, J. C., Wolf, B. C. \& Neiman, R. S. The pathogenesis of splenic extramedullary hematopoiesis in metastatic carcinoma. Cancer 63, 1539-1543 (1989).

3. Wolf, B. C. \& Neiman, R. S. Hypothesis: splenic filtration and the pathogenesis of extramedullary hematopoiesis in agnogenic myeloid metaplasia. Hematol. Pathol. 1, 77-80 (1987).
4. Yamamoto, K. et al. Extramedullary hematopoiesis: elucidating the function of the hematopoietic stem cell niche (review). Mol. Med. Rep. 13, 587-591 (2016).

5. Konoplev, S., Hsieh, P. P., Chang, C. C., Medeiros, L. J. \& Lin, P. Janus kinase 2 V617F mutation is detectable in spleen of patients with chronic myeloproliferative diseases suggesting a malignant nature of splenic extramedullary hematopoiesis. Hum. Pathol. 38, 1760-1763 (2007)

6. Mesa, R. A., Li, C. Y., Schroeder, G. \& Tefferi, A. Clinical correlates of splenic histopathology and splenic karyotype in myelofibrosis with myeloid metaplasia. Blood 97, 3665-3667 (2001).

7. Oguro, $\mathrm{H}$. et al. 27-Hydroxycholesterol induces hematopoietic stem cell mobilization and extramedullary hematopoiesis during pregnancy. J. Clin. Invest. 127, 3392-3401 (2017).

8. Zherebitskiy, V., Morales, C. \& Del Bigio, M. R. Extramedullary hematopoiesis involving the central nervous system and surrounding structures. Hum. Pathol. 42, 1524-1530 (2011).

9. Rabischong, B., Larrain, D., Charpy, C., Dechelotte, P. J. \& Mage, G. Extramedullary hematopoiesis and myeloid metaplasia of the ovaries and tubes in a patient with myelofibrosis: case report and concise review of the reported cases. J. Clin. Oncol. 28, e511-e512 (2010).

10. Fraga, G. R. \& Caughron, S. K. Cutaneous myelofibrosis with JAK2 V617F mutation: metastasis, not merely extramedullary hematopoiesis! Am. J. Dermatopathol. 32, 727-730 (2010).

11. Bowling, M. R. et al. Pulmonary extramedullary hematopoiesis. J. Thorac Imaging 23, 138-141 (2008).

12. Pipoly, G. M. \& Rogers, J. Cardiac tamponade resulting from pericardial extramedullary hematopoiesis: a case report and review of the literature. Cancer 44, 1504-1506 (1979).

13. Lavu, S. \& Tefferi, A. Cardiac tamponade in myelofibrosis: a Mayo clinic series of nine consecutive cases. Am. J. Hematol. 92, E544-E545 (2017).

14. Khan, A., Milley, P. S., Spaulding, M. B. \& Marchetta, F. C. An unusual cause of cervical adenopathy. Extramedullary hematopoiesis. Arch. Otolaryngol. 108, 523-524 (1982)

15. Koch, C. A., Li, C. Y., Mesa, R. A. \& Tefferi, A. Nonhepatosplenic extramedullary hematopoiesis: associated diseases, pathology, clinical course, and treatment. Mayo Clin. Proc. 78, 1223-1233 (2003). 